\title{
On the failure of fowl to adapt to prism induced displacement: Some theoretical analyses
}

\author{
MARTIN B. ARLIKSKY \\ UNIVERSITY OF KANSAS
}

Results of perceptual research involving certain species of fowl have prompted the conclusion that fundamentally unique visual-perceptual processes are operative in animals of this type. These findings have also been used as the basis for nativistic arguments conceming the genesis of visual space perception. The methodological and experimental bases for the se conclusions are examined, with reference to studies which have employed angularly deviating wedge prisms to transform the visual field. It is proposed that conclusions drawn from previous studies are unwarranted, for the experimental situations did not provide sufficient conditions whereby the animals could change or modify their responses. Suggestions for further research are offered in terms of perceptual learning.

When certain species of fowl (e.g., hens and chickens of various ages) are fitted with image displacing wedge prisms, they do not adapt to the induced image shift (Pfister, 1955; Hess, 1956).

The experimental results are widely cited, usually in conjunction with proposals concerning the genesis and development of visual space perception. The basic statement usually takes the following form: "Apparently the innate picture which the chick has of the location of objects in its visual world cannot be modified through learning if what is required is that the chick learns to perform a response which is antagonistic to its instinctive one" (Hess, 1956, p. 78; italics supplied).

Gregory (1966), while accepting the conclusion of nonadaptation in chicks, nevertheless makes the following point (p. 209): "It seems quite clear from the various experiments that animals show far less adaptation to shift or reversal of the image than do human observers. Indeed, only monkeys show any adaptation at all" (italics supplied). The implication of phylogenetic distinction is basic to the present analyses and is developed as follows.

The higher primates have the structural anatomy and neurophysiology by which to correct disrupted visuomotor (e.g., eye-hand) coordination. Consequently, possessing this source of "discrepancy feedback," primates adapt in much the same way as do their human counterparts (e.g., Bossom, 1964; Bossom \& Hamilton, 1963; Sperry, 1964). Quite the opposite situation exists in the phylogenetically lower fowl.

For instance under ordinary circumstances, where chickens (or newborn chicks) peck, they do not secure the intended morsel with the claws prior to or during the pecking. They do, of course, scratch and dig for food, but this appears to be quite distinct from the visuo- motor coordination involved with the actual peckingingestion sequence. An eye-beak analogy (i.e., to the eye-hand coordinate system of the primate) does not appear to be valid, for even should the animal be able to view his beak through the prisms, its spatial position is determined by the rotation of the head. Thus, the necessary prism induced discrepancy and its consequent informative potential would be lacking. When fitted with $7^{\circ}(12.18 \Delta)$ angularly displacing prisms, the chicks consistently peck with a $7^{\circ}$ lateral error, depending on base orientation. Under these conditions the animals do not "adapt," i.e., they do not ultimately correct for the $7^{\circ}$ displacement induced by the prisms. Two important questions at this point are: (a) how could a newborn chick or adult chicken modify its response, and (b) why should a newborn chick change its response? The following brief analyses and discussion have reference to these two questions.

While viewing the environment through laterally displacing prisms, $O$ 's visual perception is literally "what it is." There exists no "real" or objective spatial localization of the target(s) within $O$ 's visual field. Under inspection conditions the eyes will never reconverge to focus on the undisplaced target simply because the angular deviating power of the prism determines eye position. That is, under these conditions and with respect to vision, a (separate) non-displaced target does not exist.

Correction of disrupted bisensory coordination(e.g., eye-hand type), however, requires discrepancy information from the more mobile and flexible receptor. The term "discrepancy" implies a process of veridical comparison. In this case and under proper conditions, the process involves both the relatively stable, apparent visual localization in space and an extremely modifiable trial and error sense of felt position (see Harris, 1965, for a comprehensive analysis of similar problems). Under conditions of drive or motivation, the relatively labile osteo-kinesthetic responses, determined by the very nature of the situation, "search for the objective stimulus." This behavior serves to reduce the visualkinesthetic information discrepancy.

It may be the case that without a source of tactual or other "discrepancy" feedback, newborn or adult fow1 have no information by which to proceed to correction. A discussion of potential cognitive factors is beyond the scope of this paper; however, one generally would not imply that such lower species Ss have the "knowledge" that they are being experimentally foiled. Nevertheless it is quite possible that this "expectation" could be conditioned. For instance, the average pecking error (i.e.. due to neurophysiological limits of the system) could be 
calculated. Then, lower strength prisms, with a power approximately equal to the average pecking error value, would be fitted to the animal. As the chicken pecks at the apparent image of the target it will hit the physical stimulus (kernel of corn) a certain number of times by chance. While the tactual analog has not been attained, the animal is provided with the experience of pecking under two reinforcement conditions. Thus, after the chick has pecked under reward conditions, subsequent pecks under non-reward conditions may motivate the animal to a series of "searching" behaviors. Obviously, potential difficulties concerning partial reinforcement effects (e.g., variable ratio contingencies) would require control.

The peck-reward associative sequence is perhaps more relevant in the case of newborn chicks, (question (b) above). That is, while the pecking response does appear to be innate (unlearned), the reception of food does not. The chick presumably has had no pre-hatch pecking experience. Consequently, upon hatching, there is no reason to expect that reinforcement as such, has any immediate potential to modify the animal's behavior, i.e., upon being hatched the chick pecks irrespective of reinforcement conditions, else experiments such as Hess' (1956) would not be possible.

However, after a certain period of responding under these reinforcement conditions, the animal may actually learn the peck-no reward cont ingency, simply because there have been no other reinforcement conditions in its experience. Adaptive behavior in this case, would be confounded by an uncontrolled learning sequence.

The possibility remains of another, more direct means of providing "corrective discrepancy feedback." Again, the basic technique would consist of a simple associative learning sequence. In attempting to provide for the necessary feedback, one would include prism strength as a variable in a modified factorial design.

With two levels of prism strength, another possible variable, training might be included. The first level would consist of pretraining to peck and receive a buzzer and/or light which would emanate from the exact spatial location of the food, and be presented simultaneously with the pecking-ingestion reinforcement sequence. The second level would have no pretraining and create, in effect, an instrumental conditioning situation. Among other conditions of testing for adaptation, the pretraining reinforcement buzzer might be presented whenever the chick pecked and would emanate from the kernel as in training. Or, perhaps, the frequency of the stimulus could be varied, becoming more similar to the original tone as the animal approached the target and vice versa. Newborn chicks with prism strength equal to the average pecking error and in the "instrumental" learning condition would perhaps yield the higher level of learning. Careful control might allow some pretraining for newborn chicks; however, pretrained adult chickens under both levels of prism strength would presumably show the higher degree of learning. Also, a prism strength by training by age interaction might be deducible, since adult animals, having had more prior reinforcement experience, would presumably correct (e.g.) a $1^{0}$ displacement more easily than one of $7^{\circ}$. However, these remain empirical questions. (A recent study by Pick \& Hay, 1966, contains data on the temporal course of eye-ear adaptation in human Ss and should be consulted for further reference.) Other relevant variables immediately suggest themselves, such as drive level. Finally, perhaps instrumentation could be devised whereby fowl are conditioned to use the leg-claw appendage during the food reinforcement sequence. Presumably, this would provide for a source of tactual-motor feedback upon testing for adaptation.

Concerning the possibility of visual adaptation, i.e., adaptation under inspection conditions, the following studies deserve mention. Under certain conditions human Os show adaptation when apparently the main source of feedback is oculomotor (McLaughlin, Rifkin, \& Webster, 1966). Similar results are reported by Rock, Goldberg, \& Mack (1966) and are assumed to involve the conflict between information derived from the structure of the scene and information derived from eye position. However, problems arise concerning (a) crossspecies response measurement, and (b) differences in the structural neuroanatomy of the fowl visual system, and appear to preclude any simple application of these findings to the studies with chicks. The original papers should be consulted for further specific reference.

If the above analysis accurately indicates the status of research involving space perception in fowl, it is apparent that the original nativistic conclusions are, at the very least, open to question.

\section{References}

Bossom, J. Mechanisms of prism adaptation in normal monkeys. Psychon. Sci., 1964, 1, 377-378.

Bossom, J., \& Hamilton, C. R. Interocular transfer of prism altered coordinations in split-brain monkeys. J. comp. physiol. Psychol., 1963, 56, 769-774.

Gregory, R. L. Eye and brain: the psychology of seeing. N. Y. World U. Library, 1966.

Harris, S. Perceptual adaptation to inverted, reversed and displaced vision. Psychol. Rev., 1965, 72, 419-444.

Hay, J. C., \& Pick, H. L. Visual and proprioceptive adaptation to optical displacement of the visual stimulus. J. exp. Psychol., $1966,71,150-158$.

Hess, E. Space perception in the chick. Scient. American, 1956, 195 (1): $71-80$.

McLaughlin, S. C., Rifkin, K. I., Webster, R. G. Oculomotor adaptation to wedge prisms with no part of the body seen. Percept. \& Psychophys., 1966, 1, 452-458.

Pfister, H. Über das Verhalten der Hühner beim Tragen von Prismen. Unpublished doctoral dissertation, University of Innsbruck, Austria, 1955. (See also deiailed discussion in: Smith, K. U., \& Smith, W. M. Perception and motion. Philadelphia, Saunders, 1962.)

Rock, I., Goldberg, J., \& Mack, A. Immediate correction and adaptation based on viewing a prismatically displaced scene. Perception \& Psychophys., 1966, 1, $351-354$.

Sperry, R. W. The great cerebral commissure. Scient. American, $1964,210(18): 42-52$. 